\title{
System-Theoretic Trends in Econometrics
}

\author{
J. M. Schumacher \\ Centre for Mathematics and Computer Science, P.O. Box 4079, 1009 AB Amsterdam, \\ The Netherlands, and \\ Department of Economics, Tilburg University, P.O. Box 90153, 5000 LE Tilburg, The Netherlands
}

The dream of econometrics has metamorphosed into a technical problem in system theory.

(R. E. Kalman)

\begin{abstract}
This is a brief survey of some recent research trends in econometrics which make extensive use of techniques developed in system theory. In particular, we pay attention to the following subjects: cointegration, error correction, and the representation of systems; path controllability, system inversion, and trackability; inputs, outputs, and errors-in-variables.
\end{abstract}

\section{Introduction}

System theory interacts with the theory of economics and econometrics in rather diverse ways, and the past few decades have seen the arrival and sometimes also the departure of a rich variety of research trends in the interface. The story might begin with The Mechanism of Economic Systems [55], a book that was published in 1953 although it was based on notes that the author, Arnold Tustin, had written immediately after World War II. In this book, Tustin proposed to model the workings of a national economy by analog simulation using clever mechanical and electrical devices which he described in some detail. Apparently his hope, as an electrical engineer, was to use such nonlinear models to explain and remedy business cycles much in the same way as unwanted oscillatory motions in servomechanisms can be suppressed by appropriate controller design. As noted by Aoki [3], this approach doesn't seem to have had widespread influence among economists.

There have been other trends, however, which did acquire a status of permanence in the economic and econometric literature. Optimal control theory, in the style that emerged in the fifties, has found its way into the economic realm and is well and alive there. This is evidenced in recent textbooks such as [16] and [53]. Optimal stochastic control theory has found application in financial management; a recent survey is provided in [31]. There are other areas that are more or less allied to system theory and that are extensively used in economics, such as the theory of differential games, but we will leave these out of our discussion.

An example of a standard and full-fledged subject in system theory that has had an undeniable influence in econometrics is, of course, the Kalman filter. 
Its importance was recognized in the standard reference [22], and the Kalman filter can now be considered as one of the standard tools in the study of time series and dynamic economic models (cf. $[14,48]$ ). Further interaction between system theory and econometrics takes place in the field of identification. The fundamental problems that are involved here were stirred up by R.E. Kalman [30]. A recent detailed elaboration of some of the points raised by Kalman can be found in $[36,37]$. At a more technical level, the recent book by Hannan and Deistler [23] provides an excellent reference for the way that system theory and statistics interact to solve identification problems.

In this paper, we shall attempt to highlight some of the newer research trends in econometrics which make extensive use of ideas and techniques from system theory. First, we shall discuss the issue of 'cointegration' which has been heavily debated in econometric circles during the past decade. One of the central points in the discussion is a result known as the Granger representation theorem; this is basically a theorem about alternative representations for linear dynamic systems, which in system-theoretic terms would fall under the heading of realization theory (or as some would perhaps prefer to say: the theory of system representations and transformations). There is also an aspect of control in the cointegration debate; in particular, the tracking of targets is involved. The ability of a system to track a given target is a classical subject in system theory, and recently there have been some efforts to extend this older work and to apply it in specific economic contexts. We shall briefly discuss the results in this area in Sect. 3. Our final topic will concern the selection of 'inputs' and 'outputs' ('endogenous' and 'exogenous' variables, in econometric terminology). This subject allows a four-fold decomposition brought about by the two divisions static/dynamic and deterministic/stochastic; we shall discuss all four cases, to bring out some interesting analogies. The final Sect. 5 contains concluding remarks.

In this paper we will not cover all of the impulses to the application of systemtheoretic ideas in economics that are due Aoki and his co-workers, such as the ideas concerning aggregation and reduction by balancing; instead we refer to Aoki's recent book [4]. For additional material, we also refer to the special issue of the Journal of Economic Dynamics and Control on Economic Time Series with Random Walk and Other Nonstationary Components (Vol. 12-2/3 (1988), edited by $\mathrm{M}$. Aoki), the special issues of Computers \& Mathematics with Applications on System-Theoretic Methods in Economic Modeling (Vols. 17-8/9 (1989) and 18-6/7 (1989), edited by S. Mittnik), and the survey paper by E.J. Moore [38].

\section{Cointegration, Error Correction, and the Representation of Systems}

Many economic time-series show an apparent random drift, which may be explained by a lack of forces which tend to drive the variable under study to some preferred level. Since the traditional econometric methods of dealing with 
time-series are based on stationarity assumptions, it is standard practice (recommended for instance in [5]) to pre-filter the data by taking differences. Differencing once will reduce a 'random walk'-like behavior to stationarity. If necessary, a time-series may be differenced several times in order to achieve stationarity. A scalar time series is said to be integrated of order $d$ if it reaches stationary after differencing $d$ times. Since there is loss of information involved in taking difference (a differenced model can only describe relations between changes of variables, not relations between the absolute levels), over-differencing should be avoided.

In the context of vector time series, clearly there may be different orders of observation between the components of the vector; more generally, it can happen that certain linear combinations of the components have lower order of integration than the components themselves. This may be seen as strong evidence for the presence of economic forces which tend to keep a certain balance between the components, and the discovery of such relations is therefore of considerable interest. Examples are the relations between consumption and income and between short-term and long-term interest rates $[9,13]$. Generally speaking, cointegration is found in so-called error correction models. Suppose that we have two (vector) variables $y_{t}$ and $z_{t}$ which tend to satisfy a static 'target' relation

$$
A y_{t}+B z_{t}=0
$$

The presence of this target relation can be reconciled with the presence of (first-order) nonstationary dynamics by specifying an 'error correction' model:

$$
A_{1}(L) \Delta y_{t}+B_{1}(L) \Delta z_{t}+D(L)\left[A y_{t-1}+B z_{t-1}\right]=C(L) \varepsilon_{t}
$$

(The notation here is the econometric one: $L$ is the lag operator that maps $\left(x_{t}\right)_{t}$ to $\left(x_{t-1}\right)_{t} ; \Delta=I-L$ is the difference operator, which maps $\left(x_{t}\right)_{t}$ to $\left(x_{t}-x_{t-1}\right)_{t}$; $A_{1}(z), B_{1}(z), D(z)$, and $C(z)$ are polynomial matrices; $\left(\varepsilon_{t}\right)_{t}$ is white noise.) This way of incorporating long-term dynamics into short-term dynamic models originates in $[9,47]$.

A precise formulation of the connection between cointegrated models and error correction models has been proposed by C.W.J. Granger in an unpublished manuscript [20] and in the paper [13]. Specifically, Granger calls a process $\left(x_{t}\right)_{t}$ cointegrated of order $d, b$ if all components are integrated to order $d$, and if some nontrivial linear combination $z_{t}=\alpha^{\prime} x_{t}$ is integrated of order $d-b$ where $b>0$. A process $x_{t}$ in $\mathbf{R}^{n}$ that is cointegrated of order 1,1 is said to have cointegrating rank $r$ if $\alpha^{\prime} x_{t}$ is stationary for some $r \times n$-matrix $\alpha^{\prime}$ of full row rank, and if $\beta^{\prime} x_{t}$ is nonstationary for any matrix $\beta^{\prime}$ whose rank exceeds $r$. The Granger representation theorem gives the connection between representations of 'autoregressive' and 'moving-average' type for time series that are cointegrated of order 1,1 . The following version uses a formulation proposed by Johansen [26].

The Granger Representation Theorem. Assume that the $\mathbf{R}^{n}$-valued process $\left(x_{t}\right)_{t}$ satisfies

$$
\Delta x_{t}=C(L) \varepsilon_{t}
$$


where $\left(\varepsilon_{t}\right)_{t}$ is zero-mean white noise of unit variance, and $C(z)$ is an $n \times n$ matrixvalued function that is holomorphic on the disk $|z|<1+\rho$ and that is nonsingular on the same disk except at 1 , where $C(1)$ has rank $n-r$. Let $\alpha$ and $\beta$ be $n \times r$ matrices of full column rank such that $\alpha^{\prime} C(1)=0$ and $C(1) \beta=0$. If the $r \times r$ matrix $\alpha^{\prime}(d C / d z(1)) \beta$ is nonsingular, then the process $\left(x_{t}\right)_{t}$ is cointegrated of order 1,1 with cointegrating rank $r$ and satisfies the equation

$$
\Pi_{0} x_{t}+\Pi_{1}(L) \Delta x_{t}=\varepsilon_{t}
$$

where

$$
\Pi_{0}=\beta\left(\alpha^{\prime}(d C / d z(1)) \beta\right)^{-1} \alpha^{\prime}
$$

The processes $\left(\Delta x_{t}\right)_{t}$ and $\left(\alpha^{\prime} x_{t}\right)_{t}$ are stationary so that the representation (2) may be seen as an error correction representation.

Conversely, suppose the process $\left(x_{t}\right)_{t}$ satisfies an equation (2) where $\left(\varepsilon_{t}\right)_{t}$ is white noise and where the matrix function $\Pi(z)=\Pi_{0}(z)+(1-z) \Pi_{1}(z)$ is holomorphic and nonsingular on the disk $|z|<1+\rho$ except at $z=1$ where $\Pi_{0}=\Pi(1)$ has rank $r$. Let $\alpha$ and $\beta$ be $n \times(n-r)$-matrices of full column rank such that $\alpha^{\prime} \pi_{0}=0$ and $\pi_{0} \beta=0$. If the $(n-r) \times(n-r)$-matrix $\alpha^{\prime} \Pi_{1}(1) \beta$ is invertible, then the process $\left(x_{t}\right)_{t}$ is cointegrated of order 1,1 with cointegrating rank $r$ and satisfies an equation (1) in which $C(z)$ is holomorphic and nonsingular in the disk $|z|<1+\rho$ except at the point $z=1$, where

$$
C(1)=\beta\left(\alpha^{\prime} \Pi_{1}(1) \beta\right)^{-1} \alpha^{\prime}
$$

The proof of the Granger representation theorem in [13] is somewhat hard to follow. Engle sketches a different proof, due to B.S. Yoo, in [12]. This proof is based on what Engle calls the Smith-McMillan-Yoo form; it is actually a Smith form with respect to the ring of causal stable rational functions. In [26], Johansen uses the context of functions that are holomorphic on an open disk containing the unit circle (which is more general than the rational context used by Yoo), and he provides a third proof. Apparently it hasn't been noticed in this literature that essentially a matrix generalization is involved here of the following simple rule from complex function theory: if $f(z)$ is holomorphic in a neighborhood of $z_{0}$, then $f^{-1}(z)$ has a simple pole at $z_{0}$ if and only if $d f / d z\left(z_{0}\right)$ is nonzero, and in that case the residue of $f^{-1}(z)$ at $z_{0}$ (i.e. the coefficient of $\left(z-z_{0}\right)^{-1}$ in the Laurent series development of $f^{-1}(z)$ around $\left.z_{0}\right)$ is given by $\left(d f / d z\left(z_{0}\right)\right)^{-1}$. In the matrix case, one has to take directions into account, and the resulting residue formula is given below. We shall say that a matrix function $G(z)$ has a simple pole at a point $z_{0}$ of the complex plane if $G(z)$ has a pole at $z_{0}$ but $\left(z-z_{0}\right) G\left(z_{0}\right)$ doesn't have a pole there.

Residue Formula. Let $F(z)$ be $n \times n$ matrix function that is holomorphic in a neighborhood of $z_{0}$, and suppose that $F(z)$ is nonsingular in a neighborhood of $z_{0}$ except at $z_{0}$ itself. Let the rank of $F\left(z_{0}\right)$ be $n-r$; let $\alpha$ and $\beta$ be $n \times r$-matrices of full column rank such that $\alpha^{\prime} F\left(z_{0}\right)=0$ and $F\left(z_{0}\right) \beta=0$. Under these conditions, the matrix function $F^{-1}(z)$ has a simple pole at $z_{0}$ if and only if the constant 
matrix $\alpha^{\prime}\left(d F / d z\left(z_{0}\right)\right) \beta$ is invertible, and in that case the residue of $F^{-1}(z)$ at $z_{0}$ has rank $r$ and is given by

$$
\operatorname{Res}\left(F^{-1}(z) ; z_{0}\right)=\beta\left(\alpha^{\prime}\left(d F / d z\left(z_{0}\right)\right) \beta\right)^{-1} \alpha^{\prime}
$$

The formula is given by Lancaster for the case in which $F(z)$ is a polynomial matrix [35, pp. 60-65]; the holomorphic version is formula (4.18) in [50]. The proof is based on a suitable ('local') version of the Smith form. To see how the residue formula applies to the Granger representation theorem, we note that $\Pi(L)$ and $C(L)$ should be related by

$$
\Pi(L) C(L)=\Delta
$$

This means that

$$
C^{-1}(z)=\Pi_{0}(1-z)^{-1}+\Pi_{1}(z)
$$

so that $\Pi_{0}$ is the residue of $C^{-1}(z)$ at 1 , and of course we also have

$$
\Pi^{-1}(z)=C(1)(1-z)^{-1}+(C(z)-C(1)) /(1-z)
$$

so that $C(1)$ is the residue of $\Pi^{-1}(z)$ at 1 .

Aside from the technicalities, a more fundamental point that might be brought up in connection with the Granger representation theorem is the following. The theorem purports to be a statement about different representations of the same thing, but it is actually not too clear what it is that is being represented. Statements about equivalence of representations are traditionally formulated in situations in which there is a unique stationary solution associated with each representation, and in this case there is of course no problem - what is represented is that stationary solution. If one leaves the domain of stationary series, however (as one is forced to do in order to discuss phenomena such as cointegration), then this obvious answer is no longer applicable. The difficulty is noted by Davidson, who writes: "In fact, because of missing constants of integration a process such as [one given by a vector autoregressive equation $\Pi(L) x_{t}=\varepsilon_{t}$, with $\Pi$ (1) singular] cannot give a complete description of the generation process of the variables; it must be understood as representing a stationary process in the differences" [8, p. 8/9]. A more satisfactory approach, however, should address the problem of nonunique solutions directly. The idea of considering sets of solutions rather than individual solutions is a key point in the work of J.C. Willems $[57,58]$, which already has given rise to an extensive theory of equivalent representations for linear deterministic systems (cf. the survey [51]). It would seem that a similar theory will have to be developed for the stochastic case in order to allow for an exact and complete formulation of results such as the Granger representation theorem.

Now, let us consider briefly the general situation of higher-order cointegration. If $\left(x_{t}\right)_{t}$ is a process that is integrated of order $d>0$, then the Wold decomposition implies a representation of the form

$$
\Delta^{d} x_{t}=C(L) \varepsilon_{t}
$$


We shall continue to assume that the matrix function $C(z)$ is holomorphic on an open disk containing the unit circle and that $C(z)$ is nonsingular on the same disk except at $z=1$. It is natural to define the cointegration space of order $k$ as the set of all vectors $\alpha$ such that $\Delta^{k} \alpha^{\prime} x_{t}$ is stationary. If we denote the dimension of this space by $n_{k}$, then we may call the indices $\left(n_{0}, n_{1}, \ldots, n_{d}\right)$ the cointegration indices of the process $\left(x_{t}\right)_{t}$. In this terminology, an $\mathbf{R}^{n}$-valued process is integrated or order 1,1 with cointegrating rank $r$ if and only if its cointegration indices are $(r, n)$. The cointegration indices can be easily expressed in terms of the coefficients of the power series development of $C(z)$ around $z=1$ : writing

$$
C(z)=\sum_{j=0}^{\infty} C_{j}(1-z)^{j},
$$

we have

$$
n_{d-i}=\operatorname{dim} \operatorname{ker}\left[C_{0} \quad C_{1} \cdots C_{i-1}\right]^{\prime}
$$

The important point to note is that the cointegration indices are not in any one-one relation with the orders of the zeros at 1 of the matrix function $C(z)$. (We recall that a nonsingular meromorphic matrix function $F(z)$ allows, with respect to a given $z_{0} \in \mathbf{C}$, a 'local' Smith form

$$
F(z)=U(z) \operatorname{diag}\left(\left(z-z_{0}\right)^{k_{1}}, \ldots,\left(z-z_{0}\right)^{k_{n}}\right) V(z)
$$

where $U(z)$ and $V(z)$ are holomorphic in a neighborhood of $z_{0}$ and invertible at $z_{0}$. The integers $k_{1}, \ldots, k_{n}$ are called the order of the zeros of $F(z)$ at the point $z_{0}$.) This is seen most clearly by comparing the formula

$$
n_{d-j}=\operatorname{dim}\left\{\alpha \mid(1-z)^{-j} C^{\prime}(z) \alpha \in H(1)\right\},
$$

in which we use the notation $H(1)$ for the space of vector functions that are holomorphic in a neighborhood of 1 , with the following formula (adapted from [41]) for the number $v_{j}$ of zeros at 1 of $C(z)$ of order $\geqq j$ :

$$
v_{j}=\operatorname{dim}\left\{\alpha(1) \mid \alpha(z) \in H(1),(1-z)^{-j} C^{\prime}(z) \alpha(z) \in H(1)\right\}
$$

Clearly we have

$$
n_{d-j} \leqq v_{j}
$$

but equality does not hold in general, as can be seen from simple examples. The most important exception to this is, of course, the case of first-order integration.

It can easily be seen that the vector functions $\alpha(z)$ which appear in (4) may be restricted to be vector polynomials, without impairing the validity of the statement. Therefore, if we allow cointegrating vectors to be polynomial rather than constant and change the definition of 'cointegration indices' accordingly, we do obtain a one-one relation between cointegration indices and orders of zeros at 1 . The importance of polynomial cointegrating vectors (PCIV's) has been emphasized by Yoo (cf. [12]). A slightly different approach is taken by Johansen [24]. He introduces what we have called the 'cointegration indices', 
and notes that their sum can at most be equal to the order $r$ of the zero of $\operatorname{det} C(z)$ at 1 . The case in which equality holds is referred to by Johansen as the 'balanced' case; since it is easily verified that

$$
\sum_{j=1}^{\infty} v_{j}=r
$$

we can see that this case is the one in which equality holds in (5) for each $j=1, \ldots, d$ and, moreover, $v_{j}=0$ for $j<d$. Johansen proceeds to show that, after constant row transformations which are summarized in a nonsingular matrix $T$, we can write

$$
T C(z)=\left[\begin{array}{c}
\tilde{C}_{0}(z) \\
(1-z) \tilde{C}_{1}(z) \\
\vdots \\
(1-z)^{k} \tilde{C}_{k}(z)
\end{array}\right]
$$

where, in the balanced case, the matrix $\widetilde{C}(z)=\left[\widetilde{C}_{0}^{\prime}(z) \cdots \widetilde{C}_{k}^{\prime}(z)\right]^{\prime}$ is nonsingular at 1 . We may also write this is a slightly different way:

$$
C(z)=T^{-1} \operatorname{diag}\left((1-z)^{k_{1}}, \ldots,(1-z)^{k_{n}}\right) \tilde{C}(z)
$$

Comparing this with (3), we see that the balanced case is characterized by the fact that the local Smith form around $z=1$ can be obtained using only a constant transforming matrix on the left side. In general, one will have to use a nonconstant transformation; although the local Smith form in principle calls for holomorphic transformations, Johansen proves by a direct argument that a polynomial transformation on the left hand side will suffice. (In the rational case, one might appeal to the Smith-McMillan form to prove this; in fact, this is what Yoo does.) The polynomial transformation can then be interpreted as a transformation of the variables in which linear combinations are taken of contemporaneous and lagged components.

So, either by introduction of polynomial cointegrating vectors or by polynomial transformations of the variables, the structure of cointegrated systems can be studied through the zero structure of an associated matrix function at $z=1$. This may help to solve remaining problems, such as the formulation of analogs of the Granger representation theorem for higher-order cointegrated series (partial results on this can be found in [24] and [8]). Another important question is, to what extent polynomial cointegrating vectors (or polynomial transformations of the variables) are unique; the answer to this is of course critical to the discovery of 'target relations'.

In the above, we have emphasized what might be called the 'structural' aspect of cointegration. There is of course also a 'statistical' side to the matter, which is concerned with the testing of hypotheses about the cointegration structure and with the estimation of cointegrating vectors, and most of the journal literature in fact concentrates on this aspect (see for instance $[13,25,42]$ ). Virtually all of this work is concerned with first-order cointegrated systems. It 
seems, however, that even in this context there are some basic questions that remain to be answered, in particular in connection with hypothesis testing. Engle notes: "The null hypothesis of cointegration would be far more useful in empirical research than the natural null of non-cointegration. The selection of a $5 \%$ test of the non-cointegration null is very arbitrary and many researchers are assuming cointegration when these tests are only rejected at larger significance levels" $[12$, p. 26/27]. One may argue about what is natural; in a sense, the hypothesis of cointegration is the more highly structured one, and is therefore simpler and more natural. From a certain point of view, the cointegrated situation is also the more singular one, which may explain the difficulties that classical statistical methods have with adopting cointegration as the null hypothesis. Possibly the theory of zeros of matrix functions may also be of help here to unravel the singularities.

\section{The Tracking of Targets}

Although cointegration can be caused by the presence of 'common trends', another explanation that is sometimes plausible is presence of steering action. Davidson and Hendry [10] even use the word 'servo-mechanism' to describe the economic forces that keep certain variables together; Arnold Tustin would have appreciated this terminology. Error correction models are placed explicitly in a context of target following by Kloek [32]. It may then be expected that the extensive theory of tracking which has been developed in mathematical control theory should have some relevance.

There is a sizable economic literature with a clear system-theoretic motivation on the problem of exactly following a prescribed path, the so-called "path controllability'. The problem is customarily posed in a deterministic setting and bears a mathematical-economic flavor rather than an econometric one. Path controllability can be seen as an extension of Tinbergen's concept of achievability of targets in static models [54]. When the targets are solved in terms of the instruments in a static linear model, so that we have

$$
y=G u
$$

where $y$ is a vector of targets, $G$ is a constant matrix, and $u$ is a vector of instruments, then the obvious criterion for achievability of each given vector $y$ by a suitable choice of instruments $u$ is that the matrix $G$ should have full row rank. A necessary condition for this to hold is of course that the number of targets should not exceed the number of instruments; this is sometimes called the 'Tinbergen policy condition'. The dynamic version of target achievability was introduced in economics by Preston [44] and Aoki [2], after essentially the same idea had been introduced into system theory (under the name of 'functional reproducibility') by Brockett and Mesarovic [6]. In the discrete-time case, path controllability is defined to mean that, after a certain 'adjustment 
time' or 'policy lead', any given path of the target variables can be tracked exactly by proper choice of the instrument variables. The definition in the continuous-time case is slightly different, but the criterion (at least in the linear constant-parameter case) is the same: path controllability holds if and only if the transfer matrix $G(z)$ from instruments to targets has full row rank as a rational matrix $[6$, p. 559]. This is a rather attractive generalization of the static rule of Tinbergen.

Further work within the system theory community on this subject has concentrated on finding simple conditions for right invertibility in terms of the state space representation

$$
\begin{aligned}
x(k+1) & =A x(k)+B u(k), & & x(k) \in X, u(k) \in U \\
y(k) & =C x(k)+D u(k), & & y(k) \in Y
\end{aligned}
$$

A condition for right invertibility in terms of the parameters $A, B, C$, and $D$ was already given by Brockett and Mesarović, but this involved a rather big matrix formed from the parameter matrices. The following compact method for determining whether or not a system is right invertible is essentially due to Morse and Wonham [39]. Define recursively a sequence of subspaces of the state space $X$ by

$$
\begin{aligned}
T^{0} & =\{0\} \\
T^{k+1} & =\left\{x \in X \mid x=A \tilde{x}+B u \text { for some } x \in T^{k} \text { and } u \text { such that } C \tilde{x}+D u=0\right\}
\end{aligned}
$$

It is easily seen that the sequence $\left(T^{k}\right)_{k}$ is nondecreasing, and so the sequence must have a limit which is denoted by $T^{*}$. The system given by the parameters $(A, B, C, D)$ is right invertible (in the sense that the transfer matrix $G(z)=$ $C(z I-A)^{-1} B+D$ is right invertible as a rational matrix) if and only if

$$
C T^{*}+\operatorname{im} D=Y
$$

The state space framework suggests extensions to the non-constantparameter case and the nonlinear case. A characterization of path controllability for linear systems with time-varying parameters has been given by Engwerda [15]; necessarily the condition is more involved than in the constant-parameter case, but an analogy with the Morse-Wonham result can still be drawn. Necessary and sufficient conditions for (local) path controllability of discretetime nonlinear systems have been given by Nijmeijer [40], who also establishes the close relation that exists between path controllability and decouplability (the possibility of introducing a control policy in which each target is influenced by only one instrument). Recently, state space algorithms have become available to decide on the right invertibility of systems that are given in implicit form, rather than in solved form [33]. This is a return to the original formulation by Tinbergen, who starts in [54] with implicit equations rather than with a 'final form'.

One may reasonably argue that the invertibility of dynamic systems should play an important role in dynamic economic theory, simply because invertibility 
is such a basic concept; so the study of system invertibility is well-motivated. However, it is also clear that exact path following is not a realistic goal in a world full of disturbances. Alternative formulations of tracking problems can be obtained by introducing assumptions on the variables to be tracked, and a stochastic setting can be accommodated by relaxing the condition of exact tracking. For instance, Kloek in [32] assumes that the target is integrated of order 2 and requires that the tracking error should be zero-mean and weakly stationary. Situations in which some information is available about the dynamics of the signal to be followed have been studied extensively in system and control theory; in fact, this branch of control theory has its roots in the design of certain servomechanisms that were used in World War II and that took the notion of 'target' quite literally. We refer to [34, Ch. 5], [60, Ch. 6-8], [49], and [18] for a sample of the modern literature on the subject. Basically, the conclusion of these studies is that, for trackability of constants and linear trends in discretetime systems, the transfer matrix from instruments to targets should have full row rank at the point 1 of the complex plane. Moreover, if the action of the controlling mechanism is based purely on the tracking error, then it can be shown that the controller must contain what is called an 'internal model' of the signal that is to be followed.

A few remarks can be made here. Firstly, we see that again the zero structure at 1 is of importance. Secondly, a somewhat surprising conclusion is that the trackability condition is stronger than the condition for path controllability; indeed, if the transfer matrix has full row rank at some given point of the complex plane then it will certainly have full row rank as a rational matrix. This may be explained by the fact that path controllability is achieved by openloop control, whereas in the case of the trackability problem the solution is sought in the form of a closed-loop controller, which automatically adjusts the control action to changes in the signal to be followed. Thirdly, the presence of an 'internal model' might be an interesting hypothesis in situations in which control action is suspected, such as when time series are cointegrated. Structural constraints such as the one implied by the internal model principle may also be used in model specification. We note that the internal model principle has been mentioned recently by Salmon [46], who however seems to use the term to indicate compatibility between models; this is certainly a subject of interest, but not one that is related directly to the tracking problem.

The role of ideas from control theory in mathematical economics can now almost be called classical; this is true for optimal control, but also for a number of other ideas in which optimization is not necessarily involved, such as path controllability. Developments that may be expected here include further elaboration of the relation between path controllability and decoupling, and study of the structure of control policies when the instruments are in the hands of various different agents. The application of control ideas in econometric modeling is more recent and, to a considerable extent, this subject still has to take shape. In many situations in which several variables of interest are studied there is a great need for structural information to be incorporated in the specification of 
models, and the results of control theory may help to provide such information in the form of constraints that must be satisfied for control action to be effective.

\section{Inputs, Outputs, and Errors-In-Variables}

It is a generally recognized fact among econometricians that the distinction between endogenous and exogeneous variables is often debatable. For this reason (and for other reasons as well) it has been argued by J.C. Willems [56] that in a general theory of systems one should start with a notion of 'observables' or 'external variables' without imposing a priori a division between inputs and outputs. This implies that one should describe the relations between the variables in a nondiscriminating way. Having done this, one may ask which choices of inputs and outputs would be reasonable; of course, exactly what is 'reasonable' in a given situation may depend on the availability of extra information which is not expressed in the system description. We shall discuss the problem of selecting inputs and outputs in four cases, corresponding to the divisions static/ dynamic and deterministic/stochastic. The discussion will be limited to linear systems, however.

The deterministic static case would perhaps be considered trivial, but let us discuss it anyway for purposes of comparison. Suppose a linear relation between external variables $w_{i}$ is given by

$$
R w=0
$$

where we may assume that the matrix $R$ has full row rank. If we believe that it is reasonable to require that the inputs are not restricted by the equations and that the outputs are completely determined by the inputs and by the equations, then the standard procedure applies: select output variables by finding a maximal set of independent columns among the columns of $R$, name the associated components $y$, name the remaining components $u$, rewrite (6) as $R_{1} y+R_{2} u=0$ and, noting that $R_{1}$ is invertible by construction, obtain

$$
y=-R_{1}^{-1} R_{2} u
$$

which clearly has the desired characteristics. In general, the choice of inputs is not unique; however, the number of inputs is determined by the data (6). Any selection of this number of variables will 'generically' be valid as a choice of inputs.

There is a certain asymmetry in the selection procedure based on (6) since we first select the outputs and then simply let the inputs be what is left. However, if we would have represented the subspace $\operatorname{ker} R$ which effectively appears in (6) as the image rather than as the kernel of some matrix, then we would have selected the inputs first by taking a maximal set of independent rows of the representing matrix. So the seeming priority of outputs over inputs in the selection procedure above is just a consequence of the chosen representation. 
In the linear deterministic dynamic case, the problem of selecting inputs and outputs has been considered by Willems in [57]. In this case, the condition for an admissible selection of inputs and outputs might be that the transfer matrix from inputs to outputs should exist and should be proper rational. (This can be formulated more intrinsically: see [59].) The solution given in [57, 58] may be described as follows. Let a set of difference equations with constant coefficients in the variable $w(k) \in \mathbb{R}^{q}$ be given by

$$
R(\sigma) w=0,
$$

where $\sigma$ denotes the (forward) shift and $R(z)$ is a polynomial matrix which we may assume to have full row rank. The basic technique is to write $R(z)$ in the form $T(z) B(z)$ where $T(z)$ is an invertible rational matrix and $B(z)$ is 'right bicausal', i.e. $B(z)$ is proper rational and has full row rank at infinity. This factorization may be achieved by the reduction of $R(z)$ to row reduced form [27, p. 386]; indeed, note that this procedure factorizes $R(z)$ as $U(z) \Delta(z) B(z)$ where $U(z)$ is unimodular, $\Delta(z)$ is diagonal with diagonal elements of the form $z^{k}$, and $B(z)$ is right bicausal. A proposed selection of inputs and outputs will induce a partitioning of $R(z)$ as $\left[R_{1}(z) R_{2}(z)\right]$ (after possible reordering of the columns), and a corresponding partitioning of $B(z)$ as $\left[B_{1}(z) B_{2}(z)\right]$. Now, $R_{1}(z)$ will be invertible if and only if $B_{1}(z)$ is invertible, and $R_{1}^{-1}(z) R_{2}(z)=B_{1}^{-1}(z) B_{2}(z)$ will be proper rational if and only if $B_{1}(z)$ doesn't have a zero at infinity. (The 'only if' holds because $B_{1}(z)$ and $B_{2}(z)$ are coprime as matrices over the ring of proper rational functions, so there can't be a pole-zero cancellation.) The result is that the proposed selection of inputs and outputs is admissible if and only if the matrix $B_{1}(\infty)$ is non-singular. In other words, what we have to do is to select a maximal number of independent columns from the full row rank matrix $B(\infty)$-we might say that the problem is reduced to the static case.

Of course, this solution is hardly surprising to the econometrician, who is used to representing transfer matrices as quotients of matrices of polynomials in $z^{-1}$ (the backward shift). In models of the form

$$
B\left(\sigma^{-1}\right) y=A\left(\sigma^{-1}\right) u
$$

where $A(z)$ and $B(z)$ are polynomial matrices, the condition that $B(0)$ should be invertible is known as the 'causality condition'; in fact, such models are often specified with the condition $B(0)=I$ (see for instance $[22$, p. 13]).

In order to make a comparison with the stochastic situation that will be discussed below, let us see how much more difficult the problem becomes when we require that that the transfer matrix from inputs to outputs should not only be proper, but also stable. In principle, the same technique as above applies: if we can write $R(z)$ in the form $T(z) B(z)$ where $T(z)$ is an invertible rational matrix and $B(z)$ is now a proper stable rational matrix having full row rank for all $z$ with $|z| \geqq 1$, then a selection of inputs and outputs will be admissible if and only if the corresponding matrix $B_{1}(z)$ is nonsingular for all $z$ with $|z| \geqq 1$. The desired factorization of $R(z)$ can be obtained by a Wiener-Hopf factorization with respect to the unit circle [7] (cf. the interpretation of the reduction to row 
reduced form as a Wiener-Hopf factorization with respect to the point at infinity in [19]). So in this case, the input-output selection problem is essentially the following: given a matrix that is 'right unimodular' over the ring of proper stable functions, find a square submatrix that is unimodular. Obviously, this is not always possible. The simplest example would be that of a system with two variables in which neither the transfer matrix from the first to the second variable nor its inverse is stable.

Next, let us consider the stochastic case. If we suppose that both the observations and the additive noise are generated by mechanisms that can be modeled as zero-mean normally distributed variables, then the general linear model can be written as

$$
w=N x+\varepsilon
$$

where $x$ generates the observations and $\varepsilon$ is noise. The observed vector $w$ will be normally distributed with zero mean and covariance matrix $Q$, and so all observational data are summarized in $Q$. In the model (7), we could select independent rows from the matrix $N$ (which may be assumed to be of full column rank) and we might convert the model to an input-output form just as in the deterministic case. However, without further assumptions on the noise, the model (7) is hopelessly non-unique. Not even the number of inputs is well-defined; it may vary from $\mathrm{rk} Q$ (no noise) to 0 (all noise).

One possible constraint on the noise covariance matrix $\Sigma$, which is wellmotivated when the observation space $\mathbf{R}^{q}$ is considered as the Cartesian product of $q$ different one-dimensional spaces, is to require that $\Sigma$ should be diagonal. This, of course, leads to the factor analysis model, which has experienced renewed interest following Kalman's critique of the concept of identifiability in econometrics $[28,29]$. What we called 'the number of inputs' becomes 'the number of common factors' in the context of factor analysis, and it is natural to define this number as the minimal length of the vector $x$ for which a representation of the form (7) (with $\operatorname{cov}\left(\varepsilon \varepsilon^{T}\right)$ diagonal) is possible. In contrast to the unconstrained case, this number is now well-determined, but unfortunately its determination is an open problem.

From the point of view of selecting inputs and outputs, it may be more natural to think of $\mathbf{R}^{q}$ not as the product of $q$ one-dimensional spaces, but as the product of an input space and an output space (yet to be determined). A possible constraint to impose would be that the noise covariance matrix should be block diagonal corresponding to this decomposition. This leads to an alternative interpretation of the vector $x$, since it can be shown that the model

$$
\left(\begin{array}{l}
y \\
u
\end{array}\right)=\left(\begin{array}{l}
H_{1} \\
H_{2}
\end{array}\right) x+\left(\begin{array}{l}
\varepsilon_{1} \\
\varepsilon_{2}
\end{array}\right)
$$

(with $x, \varepsilon_{1}$, and $\varepsilon_{2}$ independent) holds if and only if $y$ and $u$ are conditionally independent given $x$. The conditional independence property is also used to define the notion of 'state' in stochastic systems (see for instance [52]), and so 
the problem of constructing a model of the form (8) for a given decomposition of $w$ into components $u$ and $y$ is sometimes called a realization problem $[17,45]$.

Let us say that a decomposition of $w$ into inputs $u$ and outputs $y$ is 'admissible' if there is a model of the form (8) in which $x$ has minimal length among all models of the same type corresponding to the same decomposition, and in which the matrix $\mathrm{H}_{2}$ is invertible. The invertibility of $\mathrm{H}_{2}$ will allow the model to be rewritten in an input-output form:

$$
\begin{aligned}
& \hat{y}=H_{1} H_{2}^{-1} \hat{u} \\
& y=\hat{y}+\varepsilon_{1} \\
& u=\hat{u}+\varepsilon_{2}
\end{aligned}
$$

This is the errors-in-variables form (see for instance [11]). The decomposition of $w$ into $y$ and $u$ leads to a partitioning of the covariance matrix $Q_{w w}$ :

$$
Q_{w w}=\left(\begin{array}{ll}
Q_{y y} & Q_{y u} \\
Q_{u y} & Q_{u u}
\end{array}\right)
$$

We claim: the decomposition of $w$ into inputs $u$ and outputs $y$ is admissible if and only if the matrix $Q_{y u}$ has full column rank. To see this, assume first that we do have an admissible decomposition and let (8) be a corresponding model. Because $x$ has minimum length, the covariance matrix $Q_{x x}$ of $x$ must be nonsingular. We have $H_{1}=Q_{y x} Q_{x x}^{-1}$ since obviously $H_{1} x$ is the least-squares estimate of $y$ given $x$, and likewise $H_{2}=Q_{u x} Q_{x x}^{-1}$. Because of the mutual independence of $x, \varepsilon_{1}$, and $\varepsilon_{2}$, one has

$$
Q_{y u}=H_{1} E\left[x x^{T}\right] H_{2}^{T}=Q_{y x} Q_{x x}^{-1} Q_{x u}
$$

Now, it is shown in [17] that the length of $x$ in a minimal representation of the form (8) is equal to the rank of $Q_{y u}$. From the formula above, we see that this implies that $Q_{x u}$ is surjective (and hence invertible) and that $Q_{y x}$ is injective. But then $Q_{y u}$ is injective too, by the same formula. Conversely, if it is given that $Q_{y u}$ has full column rank, then the construction of [17] immediately leads to a representation of the desired form.

The conclusion must be that imposing that the error covariance matrix should be block diagonal doesn't help very much in the selection of inputs and outputs. In particular, it doesn't rule out the possibility of attributing all observed variation to noise.

Before turning to the dynamic case, let us note that the error-in-variables model is not uniquely determined even if we fix the choice of inputs and outputs. It is easy to see that all possible solutions can be parametrized in terms of the 'true' input covariance matrix $Q$, and that all symmetric positive definite matrices $Q$ will qualify that satisfy the two inequalities

$$
Q \leqq Q_{u u}
$$

and

$$
Q_{y u} Q^{-1} Q_{u y} \leqq Q_{y y}
$$


Using the singular value decomposition, one can easily show that the latter inequality can be rewritten as a lower bound on $Q$, of the form $Q \geqq Q_{\min }$. The corresponding (non-unique) 'true' linear relation between the latent variables $\hat{y}$ and $\hat{u}$ is given by $Q_{y u} Q^{-1}$.

In the static case, several proposals have been formulated to reduce the nonuniqueness of the errors-in-variables model by bringing in some extra information; see for instance [1]. Let us see what the dynamic case has to offer. We follow the development in [21] and [43].

Our goal will be to verify the admissibility of a given decomposition of $w(t)$ in inputs $u(t)$ and outputs $y(t)$. The observational data are supposed to be summarized in a spectral density matrix $Q_{w w}(z)$ for $w$, which is partitioned according to the proposed decomposition as

$$
Q_{w w}(z)=\left(\begin{array}{ll}
Q_{y y}(z) & Q_{y u}(z) \\
Q_{u y}(z) & Q_{u u}(z)
\end{array}\right)
$$

We are looking for a 'true' transfer matrix $G(z)$ and a 'true' input spectral density $Q(z)$ which should satisfy

$$
\begin{array}{rlrl}
G(z) Q(z) & =Q_{y u}(z) \\
Q(z) & \leqq Q_{u u}(z), & |z|=1 \\
G(z) Q(z) G^{T}\left(z^{-1}\right) & \leqq Q_{y y}(z), & |z|=1
\end{array}
$$

Under suitable assumptions, the development in the static case can be followed (replace the field $\mathbf{R}$ by the field $\mathbf{R}(z)$, the partial order ' $\leqq$ ' by the partial order ' $\leqq$ pointwise for $|z|=1$ ', and the involution $M M^{T}$ by the involution $M(z)$ $M^{T}\left(z^{-1}\right)$ ). As in the static case, the set of all minimal solutions will be parametrized by the spectral density matrices $Q(z)$ that fall between an upper and a lower bound determined by the data, and the corresponding transfer matrices are then given by

$$
G(z)=Q_{y u}(z) Q^{-1}(z)
$$

However, we want to impose both causality and stationarity and so we require $G(z)$ to have all of its poles inside the unit disk. The problem is to find the restrictions on $Q(z)$ that will guarantee this property for $G(z)$.

Again, the key tool to use is the Wiener-Hopf factorization. To avoid some technical intricacies, we shall assume that both $Q(z)$ and $Q_{y u}(z)$ have constant rank on the unit circle. Then we can write

$$
Q_{y u}(z)=F_{-}(z) D(z) F_{+}(z)
$$

where

$$
D(z)=\left(\begin{array}{c}
\Delta(z) \\
0
\end{array}\right), \quad \Delta(z)=\operatorname{diag}\left(z^{\kappa_{1}}, \ldots, z^{\kappa_{m}}\right)
$$

and where $F_{-}(z)\left(F_{+}(z)\right)$ is unimodular as a matrix over the ring $\mathbf{R}_{-}(z)\left(\mathbf{R}_{+}(z)\right)$ of rational functions having all their poles inside (outside) the unit circle. (We 
also used the fact that $Q_{y u}(z)$ must have full column rank, as in the static case.) For any rational matrix $M(z)$, write $M^{*}(z)=M^{T}\left(z^{-1}\right)$; note that $M^{*}$ will be $\mathbf{R}_{-}(z)$-unimodular if $M$ is $\mathbf{R}_{+}(z)$-unimodular, and vice versa. Now, write

$$
G=F_{-} D F_{+} Q^{-1}=F_{-} D \bar{Q}\left(F_{+}^{*}\right)^{-1}
$$

where

$$
\bar{Q}=F_{+} Q^{-1} F_{+}^{*}
$$

Do a spectral factorization to write $\bar{Q}=H_{+} H_{+}^{*}$, where $H_{+}$is $\mathbf{R}_{+}(z)$-unimodular. We then have

$$
G=\left[F_{-}\right] D H_{+}\left[H_{+}^{*}\left(F_{+}^{*}\right)^{-1}\right]
$$

Because the factors between square brackets are $\mathbf{R}_{-}(z)$-unimodular, it follows that $G(z)$ will be causal and stable if and only if $D H_{+}$is causal and stable, which is the same as requiring that $\Delta H_{+}$should be causal and stable. Because $\Delta(z)$ is diagonal, this requirement entails that all entries $h_{i j}(z)$ of the $i$-th row of $H_{+}(z)$, which are rational functions having all their poles outside the unit circle, should be such that the functions $z^{\kappa_{i}} h_{i j}(z)$ have all their poles inside the unit circle. Since multiplication by a power of $z$ can only move poles between zero and infinity, we see that the $\kappa_{i}$ 's should be nonpositive and that the $h_{i j}(z)$ 's should be polynomials of degree no higher than $-\kappa_{i}$. This means that there will be no solution if one of the Wiener-Hopf indices $\kappa_{i}$ is positive, and that otherwise the solution set is parametrized by at most $\kappa$ parameters, where

$$
\kappa=-\sum_{i=1}^{i=m} \kappa_{i}
$$

(Of course, we also have the requirement that $H_{+}$should be unimodular, so the parametrization is nontrivial.)

We see that imposing the requirements of causality and stationarity may well cause a certain proposal for the selection of inputs and outputs to be rejected; if the proposed selection turns out to be admissible, then it causes the set of all possible models to be finitely parametrized. However, there is no indication how to select inputs and outputs in such a way that the associated Wiener-Hopf indices will be nonpositive; this problem was raised in [21] but apparently the question is still open. Also, as in the scalar case, the number of inputs is still undetermined and the possibility of attributing all variance to noise is not ruled out. It may be worthwhile to try out the effect of other possible constraints on the error spectral density, such as size constraints (proposed for the static case in [1]).

\section{Conclusions}

An econometrician once told me that he was amazed that system theory is still an active field, since he couldn't imagine that the analysis of the Kalman filter would not be completed by now. Apparently, the full variety of system-theoretic 
methods has as yet failed to disclose itself to the field of econometrics. System theory provides a rich set of examples which illustrate the pitfalls of modeling, and how to avoid these; Kalman has used such examples in his contributions to the ongoing debate on the fundamentals of mathematical modeling and identification. System theory also provides a large body of knowledge about state space techniques, and the applicability of such techniques to econometric problems has been shown in the work of Aoki and others. But the collection of mathematical techniques that are familiar to and developed by system theorists allows an even more intensive contact. As shown in this paper, matrix factorizations and pole-zero considerations play an important role in econometric problems, and system theorists have applied these for a long time. There is an econometric interest in representation problems, which is something about which system theory has a lot to say. The invertibility of systems is a natural concept in dynamic economic analysis, destined to play a role similar to the invertibility of matrices in static analysis; and again, system theory provides the necessary tools. While some of the questions here are no doubt more modest than the fundamental issues with which R.E. Kalman has confronted the econometric profession, they may still be a worthwhile subject for research and lead to results that will satisfy system theorists and econometricians alike.

\section{Acknowledgement}

It is a pleasure to thank Manfred Deistler, Theo Nijman, and Henk Nijmeijer for the useful information they kindly provided to me.

\section{References}

1. D.J. Aigner, C. Hsiao, A. Kapteyn, T. Wansbeek (1984). Latent variable models in econometrics. Z. Griliches, M.D. Intriligator (eds). Handbook of Econometrics, North-Holland, Amsterdam, 1321-1393

2. M. Aoki (1975). On a generalization of Tinbergen's condition in the theory of policy to dynamic models. Review of Economic Studies 42, 293-296

3. M. Aoki (1976). Optimal Control and System Theory in Dynamic Economic Analysis, NorthHolland, New-York

4. M. Aoki (1987). State Space Modeling of Time Series, Springer, Berlin

5. G.E.P. Box, G.M. Jenkins (1970). Time Series Analysis. Forecasting and Control, Holden-Day, San Francisco

6. R.W. Brockett, M.D. Mesarovic (1965). The reproducibility of multivariable systems. $J$ Math Anal Appl 11, 548-563

7. K. Clancey, I.C. Gohberg (1981). Factorization of matrix functions and singular integral operators, Birkhauser, Basel

8. J.E. Davidson (1987). Cointegration in linear dynamic systems. (Paper presented at the Econometric Society European Meeting, Copenhagen, August 1987)

9. J.E. Davidson, D.F. Hendry, F. Srba, S. Yeo (1978). Econometric modelling of the aggregate time-series relationship between consumers' expenditure and income in the United Kingdom. The Economic Journal 88, 661-692

10. J.E.H. Davidson, D.F. Hendry (1981). Interpreting economic evidence: The behavior of consumers' expenditure in the U.K. European Economic Review 16, 177-192

11. M. Deistler (1989). Symmetric modeling in system identification. H. Nijmeijer, J.M. Schumacher (eds). Three Decades of Mathematical System Theory. A Collection of Surveys at the Occasion of the 50th Birthday of Jan C. Willems, Lect Notes Contr Inform Sci 135, Springer, Berlin, 129-147 
12. R.F. Engle (1987). On the theory of cointegrated economic time series. (Paper presented at the Econometric Society European Meeting, Copenhagen, August 1987)

13. R.F. Engle, C.W.J. Granger (1987). Co-integration and error correction: Representation. estimation, and testing. Econometrica 55, 251-276

14. R.F. Engle, M. Watson (1985). Applications of Kalman filtering in econometrics. (Paper presented at the 5th World Congress of the Econometric Society, Boston, Mass., 1985)

15. J.C. Engwerda (1988). Control aspects of linear discrete time-varying systems. Int $J$ Contr $4 x$. $1631-1658$

16. G. Feichtinger, R.F. Hartl (1986). Optimale Kontrolle Oekonomischer Prozesse, De Gruyter. Berlin

17. L. Finesso, G. Picci (1984). Linear statistical models and stochastic realization theory. A Bensoussan, J.L. Lions (eds). Analysis and Optimization of Systems (Proc 6th Intern Conf An:1 Optimiz Syst, Nice, June 1984), Lect Notes Contr Inform Sci 62, Springer, Berlin, 445-470

18. B.A. Francis, M. Vidyasagar (1983). Algebraic and topological aspects of the regulator problen for lumped linear systems. Automatica 19, 87-90

19. P.A. Fuhrmann, J.C. Willems (1979). Factorization indices at infinity for rational matri . functions. Integr Eq Oper Th 2, 287-301

20. C.W.J. Granger (1983). Co-integrated variables and error-correcting models, UCSD Discussiorn Paper 83-13

21. M. Green, B.D.O. Anderson (1986). Identification of multivariable errors-in-variables model with dynamics. IEEE Trans Automat Contr AC-31, 467-471

22. E.J. Hannan (1970). Multiple Time Series, Wiley, New York

23. E.J. Hannan, M. Deistler (1988). The Statistical Theory of Linear Systems, Wiley, New York

24. S. Johansen (1988). The mathematical structure of error correction models. N.U. Prabhu (ed Statistical Inference from Stochastic Processes (Proc AMS/IMS/SIAM Joint Summer Researc Conf, August 1987), Contemporary Mathematics, Vol 80, Amer Math Soc, Providence, K I $359-386$

25. S. Johansen (1988). Statistical analysis of cointegration vectors. J Econ Dyn Contr 12, 231-254

26. S. Johansen (1989). Estimation and hypothesis testing of cointegration vectors in Gaussian vec" " autoregressive models, Preprint 3, Institute of Mathematical Statistics, Univ of Copenhagen

27. T. Kailath (1980). Linear Systems, Prentice-Hall, Englewood Cliffs, NJ

28. R.E. Kalman (1982). System identification from noisy data. A.R. Bednarek, L. Cesari (ed wo Dynamical Systems II (Univ Florida Intern Symp), Ac Press, New York

29. R.E. Kalman (1982). System identification from real data. M. Hazewinkel, A.H.G. Rinnooy K : 1 (eds). Current Developments in the Interface: Economics, Econometrics, Mathematics, ReidoDordrecht, $161-196$

30. R.E. Kalman (1983). Identifiability and modeling in econometrics. P.R. Krishnaiah (at Developments in Statistics 4, Ac Press, New York, 97-136

31. I. Karatzas (1989). Optimization problems in the theory of continuous trading. SIAM J (1,r Optimiz 27, 1221-1259

32. T. Kloek (1984). Dynamic adjustment when the target is nonstationary. International Econuma Review 25, 315-326

33. M. Kuijper, J.M. Schumacher (1990). State space formulas for transfer poles at infint (Manuscript in preparation)

34. H. Kwakernaak, R. Sivan (1972). Linear Optimal Control Systems, Wiley, New York

35. P. Lancaster (1966). Lambda-matrices and Vibrating Systems, Pergamon Press, Oxford

36. C.A. Los (1989). The prejudices of least squares, principal components and common fact * schemes. Computers Math Appl 17, 1269-1283

37. C.A. Los (1989). Identification of a linear system from inexact data: A three-variable examp Computers Math Appl 17, 1285-1304

38. E.J. Moore (1985). On system-theoretic methods and econometric modeling. Intern Econ $R_{\text {, }}$ $26,87-110$

39. A.S. Morse, W.M. Wonham (1971). Status of noninteracting control. IEEE Trans Autury Contr AC-16, 568-581

40. H. Nijmeijer (1989). On dynamic path decoupling and dynamic path controllability in econots systems. J Econ Dyn Contr 13, 21-39

41. H. Nijmeijer, J.M. Schumacher (1985). On the inherent integration structure of nonline: systems. IMA J Math Contr Inf 2, 87-107

42. P.C.B. Phillips, S.N. Durlauf (1986) Multiple time series regression with integrated proce Review of Economic Studies 53, 473-495 
43. G. Picci, S. Pinzoni (1986). A new class of dynamic models for stationary time series. S. Bittanti (ed). Time Series and Linear Systems, Lect Notes Contr Inf Sci 86, Springer, Berlin, 69-114

44. A.J. Preston (1974). A dynamic generalization of Tinbergen's theory of policy. Review of Economic Studies $41,65-74$

45. C. van Putten, J.H. van Schuppen (1983). The weak and strong Gaussian probabilistic realization problem. J Multivar Anal 13, 118-137

46. M. Salmon (1988). Error correction models, cointegration and the internal model principle. $J$ Econ Dyn Contr 12, 523-549

47. J.D. Sargan (1964). Wages and prices in the United Kingdom: A study in econometric methodology. P.E. Hart, G. Mills, J.N. Whittaker (eds). Econometric Analysis for National Economic Planning, Butterworths, London

48. W. Schneider (1986). Der Kalmanfilter als Instrument zur Diagnose und Schätzung variabler Parameter in ökonometrischen Modellen, Physica-Verlag, Heidelberg

49. J.M. Schumacher (1983). The algebraic regulator problem from the state-space point of view. Lin Alg Appl 50, 487-520

50. J.M. Schumacher (1986). Residue formulas for meromorphic matrices. C.I. Byrnes, A. Lindquist (eds). Computational and Combinatorial Methods in System Theory, North-Holland, Amsterdam, $97-111$

51. J.M. Schumacher (1989). Linear system representations. H. Nijmeijer, J.M. Schumacher (eds). Three Decades of Mathematical System Theory. A Collection of Surveys at the Occasion of the 50th Birthday of Jan C. Willems, Lect Notes Contr Inform Sci 135, Springer, Berlin, 382-408

52. J.H. van Schuppen (1989). Stochastic realization problems. H. Nijmeijer, J.M. Schumacher (eds). Three Decades of Mathematical System Theory. A Collection of Surveys at the Occasion of the 50th Birthday of J an C. Willems, Lect Notes Contr Inform Sci 135, Springer, Berlin, 480-523

53. A. Seierstad, K. Sydsaeter (1987). Optimal Control Theory with Economic Applications, Advanced Textbooks in Economics, Vol 24, North-Holland, Amsterdam

54. J. Tinbergen (1952). On the Theory of Economic Policy (2nd ed), North-Holland, Amsterdam

55. A. Tustin (1953). The Mechanism of Economic Systems. An Approach to the Problem of Economic Stabilisation from the Point of View of Control-System Engineering, Heinemann, London

56. J.C. Willems (1979). System theoretic models for the analysis of physical systems. Ricerche di Automatica 10,71-106

57. J.C. Willems (1983). Input-output and state-space representations of finite-dimensional linear time-invariant systems. Lin Alg Appl 50, 581-608

58. J.C. Willems (1986). From time series to linear system. Part I: Finite dimensional linear time invariant systems. Automatica 22, 561-580

59. J.C. Willems (1988). Models for dynamics. U. Kirchgraber, H.O. Walther (eds). Dynamics Reported (Vol 2), Wiley/Teubner, 171-269

60. W.M. Wonham (1979). Linear Multivariable Control: a Geometric Approach (2nd ed), Springer, New York 